\title{
Progress on experimental and finite element studies of oblique elastic impact
}

\author{
P. P. Garland \& R. J. Rogers \\ University of New Brunswick, Canada
}

\begin{abstract}
Oblique elastic impact of nonconforming bodies is a special case of stereomechanics that can occur in several engineering applications such as loose fitting joints, robotic tasks and granular assemblies. Of particular interest in this type of impact is the tangential force that develops between the colliding bodies due to friction and tangential compliance. The solution of this problem is made more difficult due to the constantly changing contact area over the duration of impact. These factors lead to highly nonlinear force-displacement relationship between the colliding bodies.

Analytical/numerical methods based on contact mechanics have shown the tangential force waveform experienced when spherical bodies collide at low velocities is dependent on the angle of incidence. At large angles of incidence, full sliding of the bodies will occur. In this case, the tangential force will be equal to the limiting Coulomb friction value. At smaller angles of incidence, the bodies may have varying periods of full sliding, partial slipping or complete sticking over the duration of impact. In these cases, the tangential force can be expected to oscillate during impact.

Experimental testing and finite element modeling are currently being used to verify the correctness of analytical/numerical solutions previously developed. The experimental study uses a simple pendulum with a spherical steel striker. Contact force data of the oblique impact event is collected using a single tri-axial piezoelectric force transducer. The finite element model of the impact event is coded using commercially available Abaqus/Explicit ${ }^{\mathrm{TM}}$ software. The finite element model also allows for exploration of the various stress distributions on the contact surface of the colliding bodies. Preliminary results from both methods indicate that the tangential force will oscillate for shallow angles of incidence. However, comparisons of these methods to one another and to the available solutions show significant characteristic differences.
\end{abstract}

Keywords: experimental impact, finite element analysis, contact forces, Coulomb friction, shear stress, partial-slip distribution. 


\section{Introduction}

Mechanical impact of bodies is a special case of transient contact problem which has been studied since the time of Newton. These problems are common in a number of areas of practical engineering interest including tube/support interaction, mechanical joints, fretting wear, robotic tasks and granular assemblies. Under the general classification of impact problems, there exist several cases which have been studied. These cases are characterised by some combination of material and geometric properties and initial conditions. In the discussion that follows, we limit ourselves to oblique elastic impact. For this case, the combined effects of tangential compliance and friction effects give rise to a resulting tangential force, which can oscillate for certain incidence angles within the impact duration (Maw et al. [1]). This oscillation is possible for incidence angles in which there is at least some relative sticking of coincidence points in the contact zone; for larger angles, relative sliding of coincidence points will occur.

Despite the significant body of literature available on the subject of oblique impact, the interaction of colliding bodies at the contact interface is still not well understood. The focus of the work discussed here is the development of methods that can be used to independently verify the results of a previously developed continuum model of oblique impact of identical spheres (Garland and Rogers [2]). The experimental work is aimed at verifying the overall characteristics of the contact forces, particularly the oscillation of tangential force possible at certain incidence angles, and the finite element model is aimed at verifying the stress distributions, particularly the surface shear stress component, which were assumed in developing the continuum model.

Previously developed elastic continuum models [1,3] are not as prevalent in the literature as impulse-momentum techniques or discrete model approaches. Fewer still are experimental or finite element models for this specific case of impact. In 1981, Maw et al. [4] published experimental data of spherical disks impacting a rigid flat by using an air bed apparatus. The data consisted of incidence and rebound angles collected using high speed photography. The results of this study showed favourable agreement to the numerical work, the first to show tangential force oscillation, previously published by the same authors (Reference).

Osakue and Rogers [5] published experimentally obtained contact force waveforms using a simple pendulum apparatus. Their apparatus consisted of a steel sphere attached to an aluminum pipe which, when released, would strike a steel target block that housed a tri-axial piezoelectric force transducer. The force waveforms obtained showed reasonable agreement to expected results and verified the possibility for tangential force oscillation; however, the quality of the data obtained was not sufficient for detailed comparison to judge the validity of the subsequently developed model (Garland and Rogers [2]).

Existence of a three dimensional finite element model of oblique elastic impact of identical spheres appears to absent from the literature. Lim and Stronge [6] published results of a two dimensional finite element model of 
oblique impact of cylindrical bodies, which included both elastic and plastic loading cases. The elastic loading case included in this study showed tangential force oscillation that was characteristically different from what was expected. Unfortunately, results of the stress distributions were not included with this study.

The previously developed theoretical/numerical work of our oblique impact study (Garland and Rogers [2]) is an elastic continuum model that calculates the resulting tangential force waveform from the shear stress distribution at each time step throughout the impact duration. The shear stress distribution at each time step is calculated from analytical equations which relate the shear stress over the contact zone to elastic tangential displacement of points on the surface of the colliding sphere. This model is able to reproduce all of the shear stress distributions believed to be potentially present for oblique impact cases under a Coulomb friction assumption, which include full sliding or partial-slip distributions (Johnson [7]).

The following sections discuss the progress made in developing both the experimental and finite element results for use in verifying the above mentioned elastic continuum model. These portions of our study are not complete, and so only the preliminary results are available.

\section{Experimental study}

The following section discusses the experimental apparatus, data acquisition system and the preliminary results of contact force data.

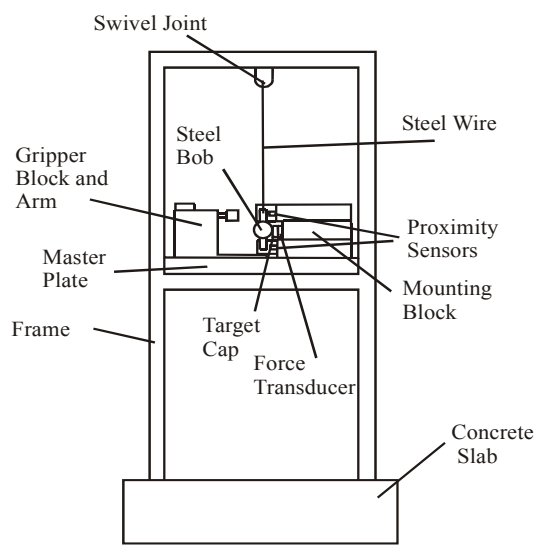

Figure 1: Schematic diagram of experimental apparatus.

\subsection{Experimental apparatus}

A schematic diagram of the experimental apparatus to be used in this study can be seen in Fig. 1. The apparatus is a simple pendulum setup with a spherical striker suspended on a steel wire. A magnetic gripper arm, which can be rotated 
to provide various horizontal incidence angles, is used to provide an initial offset of the pendulum. The mounting block is placed so that the sphere strikes the target cap at the lowest point of its trajectory. The vertical component of the force sensor is used to ensure this condition.

Upon release, the pendulum swings slightly downward and strikes a steel target cap with a spherical surface. A tri-axial piezoelectric force transducer is sandwiched between the target cap and a large steel mounting block. By varying the release distance and rotation angle of the gripper arm, various initial conditions can be set.

The contact forces during the impact will be measured using a single Kistler $^{\mathrm{TM}}$ Type 9251 piezoelectric force transducer. This sensor is able to produce three charge signals proportional to the forces applied to the face of the transducer in three directions - normal and two tangential. These charge signals are converted to the appropriate voltage values using three Kistler $^{\mathrm{TM}}$ 5010B charge amplifiers. A preload of about 10 times the maximum tangential component must be applied in the normal direction of the sensor to achieve the appropriate linear response.

For the preliminary results, the proximity sensors were not used. The exact pre-impact approach speed and incidence angle are not known. Instead, the maximum approach velocity $(\cong 100 \mathrm{~mm} / \mathrm{s})$ and approximate angles judged from the graduated scale on the master plate are used.

The data from the force transducer is collected using a National Instruments ${ }^{\mathrm{TM}}$ PCI-6110 data acquisition board. This is a 4 channel simultaneous sampling board with a resolution of 12 bits. The experimental data was collected at $5 \times 10^{6}$ points per second.

\subsection{Preliminary experimental results}

Figure 2 shows a schematic diagram of the steel sphere striking the spherical target cap at incidence as seen from a top down view. Here, we can see that the incidence angle is measured between the normal (z-axis) and the horizontal tangential ( $\mathrm{x}$-axis). From the setup, we expect all tangential loads resulting from friction to take place in the $\mathrm{x}$-axis direction.

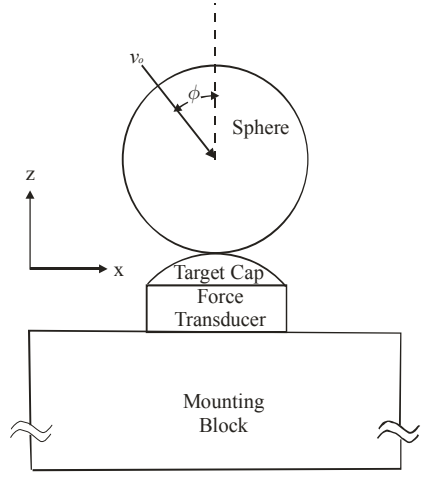

Figure 2: Configuration at incidence. 
Figures 3 (a)-(1) show the contact force data collected during typical impact experiments. For these tests, the incidence angle was varied from $10^{\circ}$ to $60^{\circ}$ in steps of $10^{\circ}$. From these graphs, we can see that the tangential force does oscillate for the more shallow angles included in the study. For the largest angle of $60^{\circ}$, the tangential force has the same shape as the normal force vector, except that its amplitude appears to be scaled by the friction effect. If we assume a Coulomb friction model with a constant friction coefficient, this result is consistent with full sliding of the contact surfaces throughout the duration of impact.
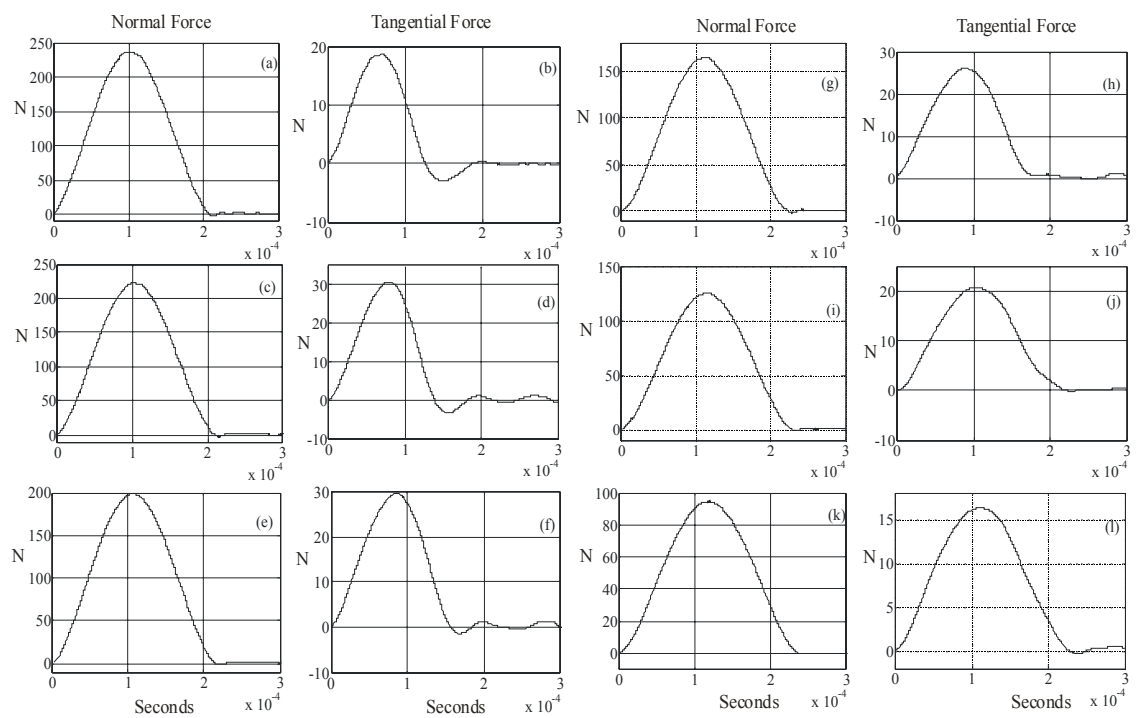

Figure 3: $\quad$ Experimental contact forces: (a) $10^{\circ}$ normal, (b) $10^{\circ}$ tangential, (c) $20^{\circ}$ normal, (d) $20^{\circ}$ tangential, (e) $30^{\circ}$ normal, (f) $30^{\circ}$ tangential (g) $40^{\circ}$ normal, (h) $40^{\circ}$ tangential, (i) $50^{\circ}$ normal, (j) $50^{\circ}$ tangential, (k) $60^{\circ}$ normal, (l) $60^{\circ}$ tangential.

During these tests, data collection continued past the end of impact, i.e., the zero crossing of the normal force vector. From results shown in Fig. 3, we can see that some post impact oscillation on both the normal and tangential force transducer signals. This oscillation is assumed to be caused by the natural system frequencies of the mounting block, and its effect appears to be more significant on the tangential force results. Since the apparently usable portion of the tangential waveform appears to extend slightly past the time of contact loss for many of the cases, it seems reasonable to assume that this ringing is affecting the tangential force results within the actual impact duration.

In order to compare these preliminary experimental results to those of the previously developed continuum model, we will normalize the tangential force waveforms and time scales by 


$$
Q=\frac{F_{x}}{\mu F_{z}} ; \quad \tau=\frac{t}{t_{\max }}
$$

where $Q$ is the normalized tangential force, $\tau$ is the normalized impact time, $F_{z}$ is the measured normal force, $F_{x}$ is the measured tangential force, $t$ is the impact time, $t_{\max }$ is the time at the loss of contact and $\mu$ is the coefficient of friction. Using the assumption of Coulomb friction and the experimental data for the $60^{\circ}$ incidence angle, the coefficient of friction is found to have a value of 0.178 .
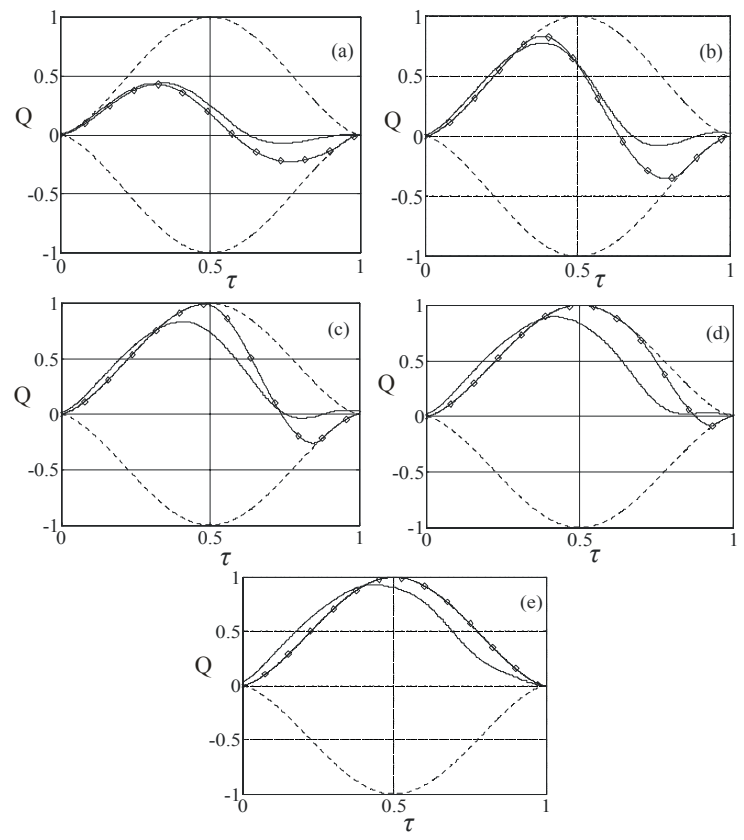

Figure 4: Normalized experimental tangential force waveforms: (a) $10^{\circ}$, (b) $20^{\circ}$, (c) $30^{\circ}$, (d) $40^{\circ}$, (e) $50^{\circ}$ (friction envelope - dashed, experimental - solid, continuum - diamond).

Figures 4 (a) through (e) show the normalized experimental tangential force results along with the normalized tangential force obtained using the continuum model (Garland and Rogers [2]) for angles ranging from $10^{\circ}$ to $50^{\circ}$, respectively. For these comparisons, the maximum experimental angle of $60^{\circ}$ has not been included because the continuum simulation indicated that full sliding begins at $50^{\circ}$.

From these graphs, we can see that the experimental results match the previous numerical results reasonably well, but do show some differences in amplitudes and times of tangential force reversal (i.e. zero crossing). Also, not all of the experimental waveforms lie completely within the idealized friction 
envelope $\left(\mu F_{z}\right)$. These differences are assumed to be related to natural system frequencies of the apparatus which act to contaminate the experimental contact force signal.

\section{Finite element model}

The following sections describe the finite element model used to simulate the spherical oblique impact problem and present preliminary results.

\subsection{Modelling considerations}

The 3-dimensional finite element model for this study has been developed in Abaqus/Explicit ${ }^{\mathrm{TM}}$ 6.6.1. We have simplified the identical sphere impact problem slightly by modelling a single deformable sphere impacting a perfectly rigid plate. From a Hertzian contact point of view, the problems are equivalent since the contact area remains circular with no distortion in the tangential plane.

The model uses an explicit dynamics approach with a coefficient of friction between the sphere and plate of 0.2 . The contact formulation for both the normal and tangential behaviour employs the penalty contact method. The model employs 10 node tetrahedral elements.

In order to provide a sufficiently large contact zone for analysis of the stress distributions on the interface, an unrealistically large velocity is provided as the initial condition to the sphere; the ratio of maximum contact zone radius to sphere radius was 0.01 , which does not violate the Hertzian contact condition. These large velocity values lead to forces which are not reasonable for the problem, and so only normalized tangential force (i.e. Eq. (1)) values are included in the results to follow.

\subsection{Preliminary finite element results}

Figures 5 (a)-(e) show the normalized finite element tangential force waveforms for incidence angles ranging from $10^{\circ}$ to $50^{\circ}$ along with those obtained from the numerical simulation (Garland and Rogers [2]). As we can see, tangential force oscillation does occur within the impact duration for certain angles of incidence. Comparison to the numerical results included in these figures shows that, once again, slight differences in the amplitudes and times of tangential force reversal are present between the two methods. The reason for these differences is unclear. One possible explanation may be the inability of the finite element model to reproduce the partial-slip shear stress distribution used in developing the continuum model.

A typical shear stress distribution along the centre line of the contact zone (i.e. line of symmetry) of the finite element model is shown in Fig. 6. This distribution corresponds to a non-dimensional time of 0.5 (i.e. half way through the impact). As can be seen, the stress distribution is plagued by significant contact noise. This raises a further mystery as to how Abaqus ${ }^{\mathrm{TM}}$ is able to produce a reasonable tangential force waveform from such noisy shear stress data. Comparison to the analytical shear stress model is not shown, but would certainly show large differences between the two methods. 
172 Computer Methods and Experimental Measurements VIII
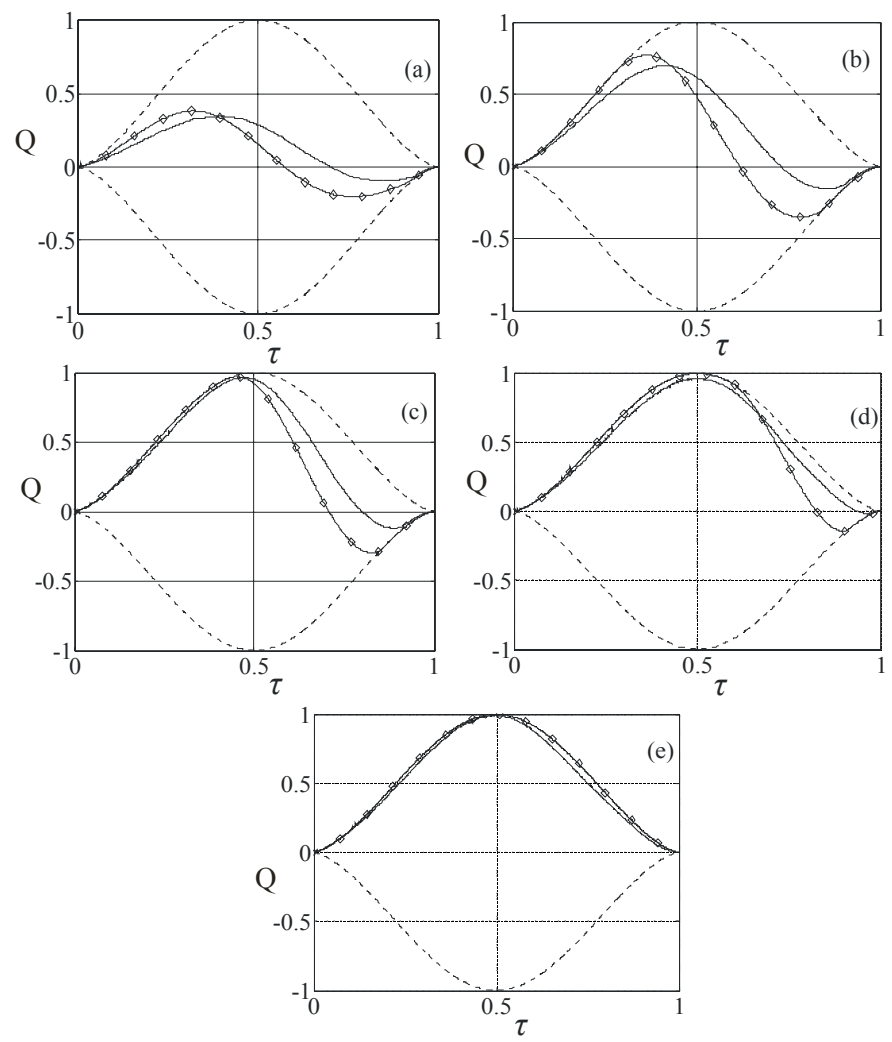

Figure 5: Normalized finite element tangential force waveforms: (a) $10^{\circ}$, (b) $20^{\circ}$, (c) $30^{\circ}$, (d) $40^{\circ}$, (e) $50^{\circ}$ (friction envelope - dashed, finite element - solid, continuum - diamond).

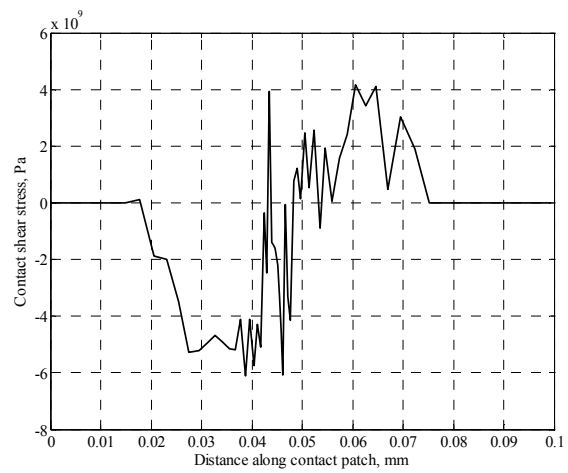

Figure 6: Typical finite element results of shear stress distribution along $\mathrm{x}-$ axis centre line of contact $\left(\phi=7^{\circ}, \tau=0.5\right)$. 
The shear stress distributions obtained from the other simulations with different incidence angles or at other times through the impact duration show similar results to those of Fig. 6. As such, use of these stress distributions in their present state will not be useful in verifying the correctness of the assumptions used in the previously developed continuum model.

\section{Conclusions and further work}

The work presented in this paper is part of a larger study of the contact forces and stress distributions that are present in the oblique elastic impact of identical spheres. The goal of the experimental and finite element modelling methods is to verify the results of a previously developed numerical model (Garland and Rogers [2]). The preliminary results obtained from these methods have shown that this verification is not possible without some further refinement of the model.

Both the experimental and finite element results have shown that, for certain angles of incidence, the tangential contact force developed during oblique impact will oscillate. The differences between the experimental and the numerical model results are believed to be caused by interference of the experimental apparatus' natural system frequencies on the force transducer's signal. The source of the differences between the finite element model and the numerical model are not clear. The differences may be related to the contact noise, they could be inherent to the finite element contact formulation method employed by the software, or they could be due to approximations in the numerical model.

At present, the experimental apparatus is being redesigned in an attempt to distribute the mounting block's natural frequencies in such a way that the effect on the force transducer is minimized. These force signals may also need to be filtered to remove or reduce the effect of these interfering frequencies.

Several alternate methods of finite element modelling are currently being explored. These include development of an implicit impact model using Abaqus/ Standard ${ }^{\mathrm{TM}}$ and exploration of other contact formulations such as Lagrange multiplier or augmented Lagrange methods. In an attempt to smooth the shear stress distribution results, the use of either time domain or spatial filtering is also being considered.

\section{References}

[1] Maw, N., Barber, J.R. \& Fawcett, J.N., The oblique impact of elastic spheres, Wear 38, pp. 101-114, 1976

[2] Garland, P.P. \& Rogers, R.J., An analytical solution for shear stress distributions during oblique impact of similar spheres, Transactions of the ASME, Journal of Computational and Nonlinear Dynamics, tentatively accepted, 2006

[3] Jaeger, J., Elastic impact with friction, PhD dissertation, Delft University, 1992 
174 Computer Methods and Experimental Measurements VIII

[4] Maw, N., Barber, J.R. \& Fawcett, J.N., The role of elastic tangential compliance in oblique impact, Transactions of the ASME, Journal of Lubrication Technology 103, pp. 74-80, 1981

[5] Osakue, E.E. \& Rogers, R.J., An experimental study of friction during planar elastic impact, Transactions of the ASME, Journal of Pressure Vessel Technology 123 (4), pp. 493- 500, 2001

[6] Lim, C.T. \& Stronge, W.J., Oblique elastic-plastic impact between rough cylinders in plane strain, International Journal of Impact Engineering Science 37, pp. 97-122, 1998

[7] Johnson, K.L., Contact Mechanics, Cambridge University Press: Cambridge, 1985 